\title{
Early detection of Alzheimer disease by means of active ageing monitoring
}

\section{Marcos Gestal}

Dementia is a brain disorder that severely affects a person's ability to carry out daily activities. Alzheimer's disease is the most common form in which dementia dementia manifests itself in people over the age of 60 .

Since there are no drugs that can stop this disease, it is important to detect it early, trying avoid (or at least delay) the accelerated cognitive decline through constant physical and mental activity.

On the other hand, the monitoring of the elderly by the public health system is a major challenge. It involves a great economic cost and consultation time.

This work shows a mobile application and a web application, to help specialists to monitor elderly people in a simple and dynamic way.

Its use should make it possible to detect patients with symptoms of cognitive impairment in their early stages. In addition, it will try to delay the arise of such symptoms, by encouraging physical and mental activity on a daily basis. 